\title{
Elusive Spin Glass Phase in the Random Field Ising Model
}

\author{
Florent Krzakala ${ }^{1,2}$, Federico Ricci-Tersenghi ${ }^{3}$ and Lenka Zdeborová ${ }^{2}$ \\ ${ }^{1}$ CNRS and ESPCI ParisTech, 10 rue Vauquelin, UMR 7083 Gulliver, Paris 75000 France \\ 2 Theoretical Division and Center for Nonlinear Studies, Los Alamos National Laboratory, NM 87545 USA \\ ${ }^{3}$ Dipartimento di Fisica, Sapienza Università di Roma, INFN - Sezione di Roma 1, \\ Statistical Mechanics and Complexity Center (SMC) - INFM - CNR, P.le Aldo Moro 2, I-00185 Roma, Italy
}

\begin{abstract}
We consider the random field Ising model and show rigorously that the spin glass susceptibility at equilibrium is always bounded by the ferromagnetic susceptibility, and therefore that no spin glass phase can be present at equilibrium out of the ferromagnetic critical line. When the magnetization is, however, fixed to values smaller than the equilibrium one, a spin glass phase can exist, as we show explicitly on the Bethe lattice.
\end{abstract}

PACS numbers: 75.10.Nr,64.60.De

Few disordered spin models have generated as much interest and studies as the Random Field Ising Model (RFIM). Yet, despite four decades of efforts in mathematics and physics, the thermodynamic properties and the nature of the phase transitions still remain debated. Originally proposed by Larkin [1] for modeling the pinning of vortices in superconductors, the RFIM has grown to be used for modeling problems as diverse as (among others) diluted antiferromagnets in a homogeneous external field [2], binary liquids in porous media [3], Coulomb -or electron- glass [4] as well as systems near the metalinsulator transition [5]. The non-equilibrium behavior of the RFIM has been used to model the physics of hysteresis and avalanches [6] and the model is also popular in the study of complex systems, for instance to model opinion dynamics [7]. The Hamiltonian of the RFIM reads

$$
\mathcal{H}=-\sum_{<i j>} J_{i j} S_{i} S_{j}+\sum_{i} h_{i} S_{i}
$$

where $J_{i j}>0$ (usually $J_{i j}=1$ ), the $N$ Ising spins $S_{i}= \pm 1$ are placed at the vertices of a graph (usually a periodic lattice), and the $\left\{h_{i}\right\}$ are quenched random fields, usually having either Gaussian distribution with zero mean and variance $H_{R}^{2}$ or a bimodal distribution $h_{i}= \pm H_{R}$.

An important controversy concerning the lower critical dimension has been resolved using rigorous argument [8, 9], and it is now known that the RFIM develops long range order for $d>2$. Another puzzle is associated to the failure of the so-called dimensional reduction property of the RFIM. Standard perturbation theory predicts to all orders that the critical behavior of the RFIM in dimension $d$ is the same as that of the pure Ising model in $d-2$ dimensions [10], a fact that violates rigorous results [9]. The reason for this failure is often related to the presence of multiple metastable states; consequently the presence or the absence of a spin glass (SG) phase in the RFIM has started to attract a lot of attention.

But is there a thermodynamic SG phase in the RFIM? Based on an extension of the RFIM to $m$-component vector spins and the large $m$ expansion [11], it has been argued that in the phase diagram of the three-dimensional RFIM the paramagnetic and ferromagnetic phases are separated by a SG phase in which the replica symmetry is broken, as in mean-field spin glasses [12]. Studies using perturbative replica field theory also show the presence of an intermediate SG phase below six dimensions [13], and other claimed that non-perturbative effect would lead to a SG phase [14]. Note that a recent work [15] uses non-perturbative renormalization group to explain the failure of the dimensional reduction without the use of replica symmetry breaking. Even in mean field models, the question has sparked debates: While in the fully connected setting of [16] no SG phase was observed, some works suggested the existence of a such a phase on the Bethe lattice [17], other do not [18], while some remained inconclusive [19]. In numerical studies, altough in [20] many solutions to the socalled naive mean-field equations have been found close to the critical temperature, results from equilibrium [21] and outequilibrium [22] Monte Carlo simulations in finite dimension found no evidences for existence of such a SG phase.

In this paper, we consider this elusive SG phase in RFIM and show rigorously that the SG susceptibility is always upper-bounded by the ferromagnetic susceptibility, for any lattice, any dimension and any choice of fields. Consequently, there cannot be a SG phase out of the critical ferromagnetic point/line. Secondly, we revise the solution of the RFIM on the Bethe lattice and show that only when the magnetization is fixed to values smaller (in absolute value) than the equilibrium one a SG phase can exist. Note that the RFIM with fixed magnetization appears in many applications involving a mapping from a lattice gas, e.g. in the Coulomb glasses or in binary liquids.

A rigorous bound on the spin glass susceptibility - A commonly accepted definition of a SG phase is the divergence of the SG susceptibility defined as

$$
\chi_{S G} \equiv \frac{1}{N} \sum_{i, j}\left(\left\langle S_{i} S_{j}\right\rangle-\left\langle S_{i}\right\rangle\left\langle S_{j}\right\rangle\right)^{2},
$$

where $\langle\cdot\rangle$ is the thermal average. The susceptibility $\chi_{S G}$ is related to the experimentally measured nonlinear susceptibility [23]. In the replica symmetry breaking theory [12] the celebrated de Almeida-Thouless condition [24] — the smallest eigenvalue of the corresponding Hessian matrix being negative - implies the divergence of this $\chi_{S G}$. The study of $\chi_{S G}$ identifies the SG transition in any theory with (static) replica symmetry breaking [32] as well as in the droplet model [25]. 
Let us now consider a RFIM on a fully connected topology with $J_{i j} \geq 0$, any other topology can be obtained by setting $J_{i j}=0$ for all pairs of spins which are not nearest neighbors. We first prove that, for any value of the external fields and on any given sample, connected correlation functions for any pair of spins $i, j$ satisfies

$$
\left\langle S_{i} S_{j}\right\rangle_{c} \equiv\left\langle S_{i} S_{j}\right\rangle-\left\langle S_{i}\right\rangle\left\langle S_{j}\right\rangle \geq 0 .
$$

In order to do so, we proceed recursively, and show that if this holds for a system with $N$ spins, then it holds for a system with $N+1$ spins. When $N=2$, we have straightforwardly $\left\langle S_{1} S_{2}\right\rangle_{c}=8 \sinh \left(2 \beta J_{12}\right) / Z^{2}$ which is indeed non-negative as long as $J_{12} \geq 0$. Consider now a system with $N$ spins $S_{i}$ with $i=1, \ldots, N$ such that $\forall i, j \in[1 \ldots N]$ Eq. (3) holds for any choice of the random fields. We add now a new spin $S_{N+1}$ with couplings $J_{(N+1) i}$ and external magnetic field $h_{N+1}$. In the $N+1$ spins system we denote $w_{ \pm}=\mathbb{P}\left[S_{N+1}= \pm 1\right]$.

We now express the correlations in the systems of $N+1$ spins in terms of the correlations in the system of $N$ spins. First, we evaluate correlations involving the new spin $S_{N+1}$ :

$$
\left\langle S_{N+1} S_{i}\right\rangle_{c}^{(N+1)}=2 w_{+} w_{-}\left(\left\langle S_{i}\right\rangle_{+}^{(N)}-\left\langle S_{i}\right\rangle_{-}^{(N)}\right),
$$

where the averages $\langle\cdot\rangle_{ \pm}^{(N)}$ are computed in the $N$ spins system whose Hamiltonian has been changed by the addition of the term $-\sum_{i} \pm J_{(N+1) i} S_{i}$, which is nothing but a change in the random fields. Given that the external fields in the measure $\langle\cdot\rangle_{-}^{(N)}$ are not greater than the corresponding fields in $\langle\cdot\rangle_{+}^{(N)}$, and that susceptibilities are non-negative in the $N$ spins system by assumption, then $\left\langle S_{i}\right\rangle_{-}^{(N)}$ is not greater than $\left\langle S_{i}\right\rangle_{+}^{(N)}$ and so we have $\left\langle S_{N+1} S_{i}\right\rangle_{c}^{(N+1)} \geq 0$.

The correlation $\left\langle S_{i} S_{j}\right\rangle_{c}^{(N+1)}$ (with $i, j \neq N+1$ ) is given by

$$
\begin{gathered}
\left\langle S_{i} S_{j}\right\rangle_{c}^{(N+1)}=w_{+}\left\langle S_{i} S_{j}\right\rangle_{c,+}^{(N)}+w_{-}\left\langle S_{i} S_{j}\right\rangle_{c,-}^{(N)}+ \\
w_{+} w_{-}\left(\left\langle S_{i}\right\rangle_{+}^{(N)}-\left\langle S_{i}\right\rangle_{-}^{(N)}\right)\left(\left\langle S_{j}\right\rangle_{+}^{(N)}-\left\langle S_{j}\right\rangle_{-}^{(N)}\right) .
\end{gathered}
$$

By the initial assumption, correlations $\left\langle S_{i} S_{j}\right\rangle_{c, \pm}^{(N)}$ are both non-negative and by the argument used above the last term is also non-negative, and so $\left\langle S_{i} S_{j}\right\rangle_{c}^{(N+1)} \geq 0$. This proves relation (3). In fact, what we have proven is a just particular case of the Fortuin, Kasteleyn and Ginibre [27] inequality, well known in mathematical physics.

Our main point is that from Eq. (3) directly follows that the SG susceptibility is upper-bounded by the ferromagnetic one:

$$
\chi_{S G}=\frac{1}{N} \sum_{i, j}\left\langle S_{i} S_{j}\right\rangle_{c}^{2} \leq \frac{1}{N} \sum_{i, j}\left\langle S_{i} S_{j}\right\rangle_{c}=\chi_{F} .
$$

This is true on any lattice and for any choice of the external fields, as long as the pairwise interactions are non-negative (and hence not frustrated). Eq. (6) implies in particular that $\chi_{S G}$ can not diverge if $\chi_{F}$ stays finite. We can say even more: The fact that the correlation matrix has all non-negative elements, $C_{i j}=\left\langle S_{i} S_{j}\right\rangle_{c} \geq 0$, implies that among all possible susceptibilities the ferromagnetic one, $\chi_{F}$, is always the largest one. This means that, in order to understand whether any kind of long range order develops in a RFIM, it is enough to check whether the ferromagnetic susceptibility is diverging, and this is a great step of reductionism! The paramagnetic phase is defined by the non-divergence of $\chi_{F}$ thus, clearly, there is no SG phase for $T>T_{c}$, where $T_{c}$ is the ferromagnetic critical temperature. This statement allows to reject many predictions in the literature: All scenarii where $\chi_{S G}$ diverges while $\chi_{F}$ is finite [11,13] are ruled out.

In the ferromagnetic phase, $T<T_{c}$, the $\chi_{F}$ would diverge because of the coexistence between the "up" and the "down" phases, with magnetization $m^{+}$and $m^{-}$respectively. Nevertheless, we can select one of these two states by using proper boundaries conditions or by adding an infinitesimal field. In each of these states the ferromagnetic susceptibility is finite (they are not critical and the clustering property holds) and therefore the SG susceptibility is again finite.

The ferromagnetic susceptibility truly diverges only exactly at a second order critical point, $T_{c}$, where two new states are generated from the paramagnetic one. At this point the Hessian, which is the inverse of the correlation matrix, develops a zero mode whose eigenvector has all non-negative elements (thanks to $C_{i j} \geq 0$ ). In other words, the two new states generated by a second order transition will have different magnetizations. The susceptibility $\chi_{F}$ is thus diverging exactly at $T_{c}$, but leads only to a ferromagnetic long-range order below $T_{c}$.

It seems to us that the only scenario, we are unable to exclude, for existence of a SG phase is to have a dense set (e.g. in $T$ ) of ferromagnetic critical points. We have, however, no reason to believe that such an exotic scenario appears in the RFIM (nor actually in any other model that we know of) and thus we conclude that there is no SG transition in the RFIM.

RFIM with fixed magnetization on the Bethe lattice - In order to go beyond the strong constraints of Eq. (6), we now consider a RFIM where the magnetization $m$ is fixed to an arbitrary value. In this case, it is worth considering the free energy $f(m)$ as a function of the magnetization $m$.

If two states exist with different magnetization, $m^{-}<m^{+}$, and if one fixes the magnetization $m \in\left(m^{-}, m^{+}\right)$, then in any finite dimension there is a phase separation between the $m^{-}$phase and the $m^{+}$phase, with the appearance of (at least) one domain wall. The free energy $f(m)$ is thus concave and given by the Maxwell construction between $m^{+}$and $m^{-}$.

Such arguments, however, do not apply when the RFIM is defined on a mean-field topology, e.g. on the Bethe lattice - a random graph with fixed coordination number, $c$. Indeed such random lattices are expanders, that is the surface-to-volume ratio of any subset of vertices does not decrease to zero when the subset is made larger (but still much smaller than the entire lattice). The shape of $f(m)$ thus does not need to be concave, and indeed we see it develops the double-well shape typical of mean field ferromagnets for $T<T_{c}$ (see Fig 11. Notice that mean-field geometries are important in many applications, such as statistical inference and combinatorial optimization (e.g. the RFIM with a fixed magnetization corresponds to the weighted graph partitioning, a well known NP-hard prob- 
lem, where the presence of a SG phase is expected).

Nonetheless the precise determination of $f(m)$ for a given sample or even for the ensemble average is a nontrivial task. In principle one would like to compute the free-energy in the presence of external field $H_{m}$ chosen such that the equilibrium magnetization is exactly $m$ and obtain $f(m)=f\left(H_{m}\right)+$ $H_{m} m$. However, for a double-well shaped $f(m)$ with minima in $m^{-}$and $m^{+}$, magnetizations in the interval $\left(m^{-}, m^{+}\right)$are in principle unreachable, as the Legendre transform computes the convex envelope of the true function $f(m)$.

We now propose an algorithm for computing $f(m)$ on the Bethe lattice even in these situations. Our approach is based on the Bethe-Peierls method, also known as cavity method [28] or belief propagation algorithm [29]. For every directed link $(i j)$ we define a cavity field $u_{i \rightarrow j}$ as the effective local magnetic field which spin $j$ receives from spin $i$. The cavity fields must satisfy the following self-consistent equations

$$
\begin{aligned}
& u_{i \rightarrow j}=\frac{1}{\beta} \tanh ^{-1}\left\{\tanh \left(\beta J_{i j}\right)\right. \\
&\left.\tanh \left[\beta\left(H_{m}+h_{i}+\sum_{k \in \partial i \backslash j} u_{k \rightarrow i}\right)\right]\right\},
\end{aligned}
$$

where the summation is over all neighbors of $i$ but $j$. The external uniform field $H_{m}$ must be chosen such as to fix the global magnetization to the desired value by

$$
m=\frac{1}{N} \sum_{i} \tanh \left[\beta\left(H_{m}+h_{i}+\sum_{j \in \partial i} u_{j \rightarrow i}\right)\right]
$$

Solved Eqs. (7, 8), the (extensive) free energy is given by

$$
\begin{aligned}
& -\beta F=\sum_{i} \log \left\{2 \cosh \left[\beta\left(H_{m}+h_{i}+\sum_{j \in \partial i} u_{j \rightarrow i}\right)\right]\right\} \\
& -\sum_{i j} \log \left\{\sum_{s= \pm 1} e^{s \beta J_{i j}} 2 \cosh \left[\beta\left(u_{j \rightarrow i}+s u_{i \rightarrow j}\right)\right]\right\} .
\end{aligned}
$$

In order to fix the magnetization to a value $m$ corresponding to the non-convex part of the free energy function $f(m)$, we solve Eqs. (7) by the following iterative scheme that forces the procedure to converge to the right fixed point, even when this is thermodynamically unstable. (A) Set $t=0$ and assign random values to $\left\{u_{i \rightarrow j}^{(0)}\right\}$ and $H_{m}$. (B) Repeat (i) compute $\left\{u_{i \rightarrow j}^{(t)}\right\}$ by Eq. (7); (ii) compute $H_{m}$ solving Eq. (8) by the bisection method; (iii) increment $t$ by 1 ; until a convergence criterion is met or a maximum number of iterations is reached. (C) If converged, compute the free energy $f(m)$ using fixed point cavity fields $\left\{u_{i \rightarrow j}^{*}\right\}$ and $H_{m}^{*}$.

Note that the bound derived in Eq. (6) is valid for any external field, and therefore for any values of $m$ such that $f(m)$ coincides with its convex envelop. This bound is, however, not valid for the values of $m$ in the interval $\left(m^{-}, m^{+}\right)$and this is the place we should check for the appearance of a SG phase. In order to do so, we study the stability of the BethePeierls solution towards the appearance of a SG order with a

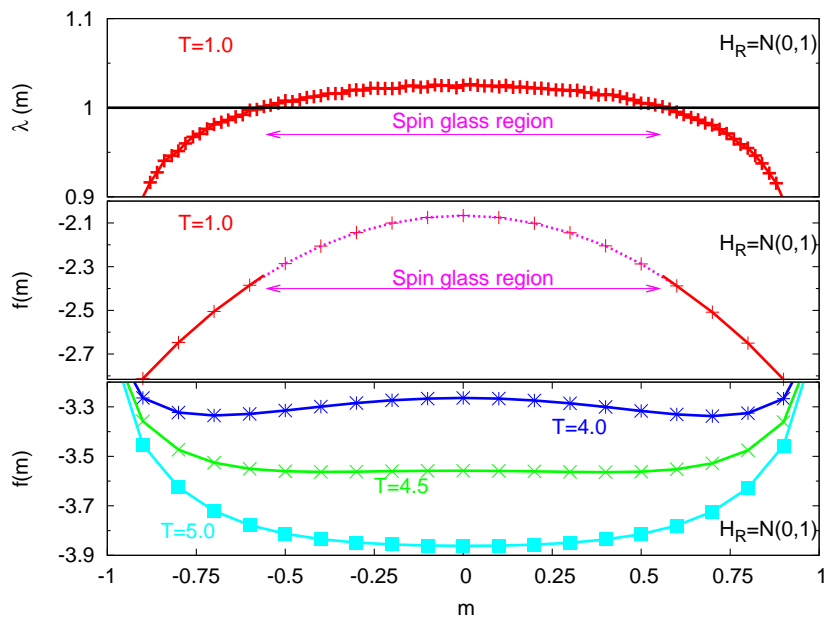

FIG. 1: (color online) Free energies $f(m)$ and the stability parameter $\lambda(m)$ versus the magnetization $m$ on a Bethe lattice of coordination $c=6$ with Gaussian random fields of unit variance and zero mean. Bottom: we show $T=5, T=4.5$ and $T=4.0$ (with $T_{c}=4.66$ ); notice the appearance of two minima for $T<T_{c}$. Middle: a low temperature case $(T=1)$. A spin glass region appears when the magnetization is fixed to low enough values. Top: the corresponding stability parameter $\lambda(m)$ showing the spin glass order for $|m|<$ 0.56 .

diverging $\chi_{S G}$. There are several different methods for computing this instability (for a review see appendix $C$ in [30]), all of them generalizing the de Almeida-Thouless condition [24]. The numerically most precise one is to study the fate of a small perturbation to the cavity fields [31], that are evolving according to the following linear equations

$$
\delta u_{i \rightarrow j}^{(t+1)}=\frac{\partial u_{i \rightarrow j}^{(t+1)}}{\partial u_{k \rightarrow i}^{(t)}} \delta u_{k \rightarrow i}^{(t)} .
$$

The divergence of the root mean square of the $\delta u$ 's signals a local instability and the appearance of a SG phase. In practice we measure the parameter $\lambda$ which is the rate of growth of the root mean square of the $\delta u$ 's.

In Fig. 1 we show a typical free energy $f(m)$ on a Bethe lattice and the stability parameter $\lambda$. We clearly see that indeed a SG phase is present for some of the non-equilibrium values of the magnetization. Fig. 2 shows the phase diagram of the RFIM on a Bethe lattice. Note that on a cubic $3 d$ lattice with Gaussian random fields, the transition in zero field is at $T_{c} \approx 4.5$ and for zero temperature at $H_{c} \approx 2.3$. Corresponding critical values on the Bethe lattice are larger, as expected for a mean field approximation. Just as in the mean field solution of [16] the ferromagnetic transition is of first order for the bimodal distribution of fields at low enough temperature [for $c \geq 4$, at zero temperature spinodal lines end in $H_{s p}=(c-1) / 2$ and $\left.H_{s f}=c-2\right]$. The SG region appears always at a smaller value of magnetization than the equilibrium one. At zero temperature the ferromagnetic critical point is also critical for the SG phase with $m=0$. In the renormaliza- 

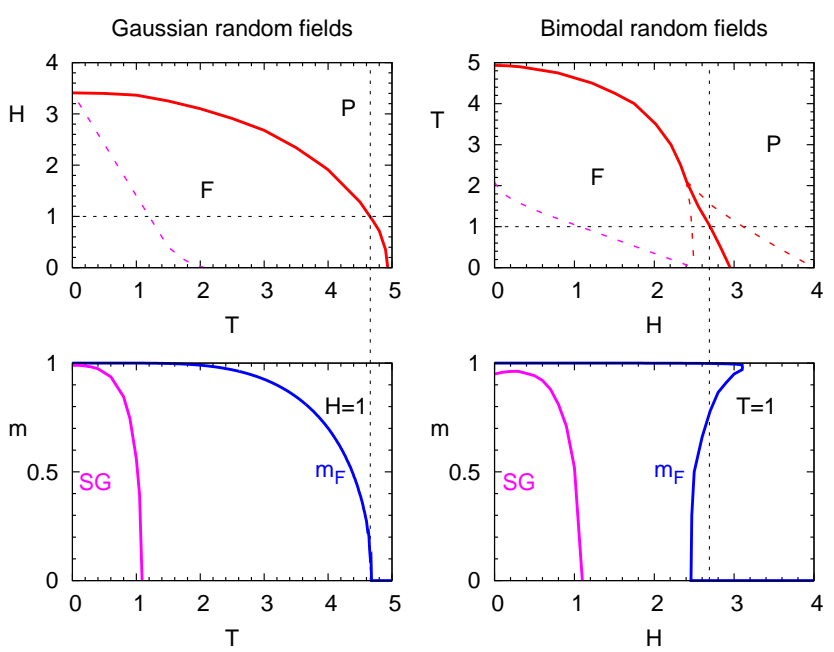

FIG. 2: (color online) Phase diagram of the RFIM on the Bethe lattice with $c=6$ with Gaussian (left) and bimodal (right) random field. Top panels: Boundary between paramagnetic (P) and ferromagnetic (F) phases (red/full line). In the low $T$ region the transition is first order for bimodal fields (red dashed line are the spinodals). Below the purple dashed line a spin glass (SG) phase exists at zero magnetization $m$. Lower panels: Equilibrium magnetization $m_{F}$ (blue/full line). A SG phase exist only for $m$ below the purple full line.

tion group approach this is the relevant fixed point and the SG instabilities seen in the perturbative approach could perhaps be linked to this fact.

Conclusions - We have shown that there is no spin glass phase at equilibrium in the RFIM, thus closing a long-standing debate on the elusive spin glass phase in this model. It is only if one fixes the magnetization to non-equilibrium values that a true SG phase can exist, as we showed explicitly on the Bethe lattice. In finite dimensional systems the existence such a stable phase, altough unlikely, remains open. This SG phase, or its vestige, although thermodynamically sub-dominant, may influence the dynamical behavior of the model. This is particularly true at zero temperature where the many local energy minima get stabilized. Our rigorous result also puts a large question mark on the field theoretical approaches that has lead to erroneous conclusions and that are still widely used in studies of more complex disordered systems such a spin glasses.

[1] A.I. Larkin, Sov. Phys. JETP 31, 784 (1970).

[2] S. Fishman and A. Aharony, J. Phys. C, L729 (1979).

[3] P.G. de Gennes, J. Phys. Chem. 88, 6469 (1984).

[4] A.L. Efros et al., J. Phys. C 8, L49 (1975).

[5] T.R. Kirkpatrick and D. Belitz, Phys. Rev. Lett. 73, 862 (1994).

[6] J.P. Sethna et al., Phys. Rev. Lett. 70, 3347 (1993). M.L. Rosinberg, G. Tarjus, F.J. Perez-Reche, arXiv:0809.3774 (2008).

[7] S. Galam, Physica A 238, 66 (1997). Q. Michard and J.-P.
Bouchaud, Eur. Phys. J. B 47, 151 (2005). F. Caccioli, S. Franz and M. Marsili, J. Stat. Mech. P07006 (2008).

[8] Y. Imry and S-K. Ma, Phys. Rev. Lett. 35, 1399 (1975).

[9] J. Bricmont and A. Kupiainen, Phys. Rev. Lett. 59, 1829 (1987).

[10] A.P. Young, J. Phys. C 10, L257 (1977). G. Parisi and N. Sourlas, Phys. Rev. Lett. 43, 744 (1979).

[11] M. Mézard and G. Parisi, J. Phys. A 23, L1229 (1990). M. Mézard and A.P. Young, Europhys. Lett. 18, 653 (1992). M. Mézard and R. Monasson, Phys. Rev. B 50, 7199 (1994).

[12] G. Parisi, J. Phys. A 13, L115 (1980). M. Mézard, G. Parisi and M.A. Virasoro, Spin Glass Theory and Beyond (World Scientific, Singapore, 1987).

[13] C. De Dominicis, H. Orland and T. Temesvari, J. Physique I 5, 987 (1995). E. Brezin and C. De Dominicis, Europhys. Lett. 44, 13 (1998); Eur. Phys. J. 19, 467 (2001). C. De Dominicis and I. Giardina, Random fields and spin glasses: a field theory approach (Cambridge University Press, 2006).

[14] G. Parisi and V.S. Dotsenko, J. Phys. A 25, 3143 (1992). V.S. Dotsenko, J. Phys. A 27, 3397 (1994).

[15] G. Tarjus, M. Tissier, Phys. Rev. B 78, 024203 (2008).

[16] T. Schneider and E. Pytte, Phys. Rev. B 15, 1519 (1977).

[17] Y. Shapir, Phys. Rev. Lett. 57, 271 (1986). R. Bruinsma, Phys. Rev. Lett. 57, 272 (1986). J.R.L. de Almeida and R. Bruinsma, Phys. Rev. B 35, 7267 (1987). A.A. Pastor, V. Dobrosavljevic and M.L. Horbach, Phys. Rev. B 66, 014413 (2002).

[18] D.J. Thouless, Phys. Rev. Lett. 57, 273 (1986). M.O. Hase, J.R.L. de Almeida and S. Salinas, Eur. Phys. J. B 47, 245 (2005).

[19] M. Bleher, J. Ruiz and V.A. Zagrebnov, J. Stat. Phys. 93, 1 (1998). T. Nowotny, H. Patzlaff and U. Behn, Phys. Rev. E 65, 016127 (2001).

[20] D. Lancaster, E. Marinari and G. Parisi, J. Phys. A 28, 3959 (1995).

[21] M.E.J. Newman and G.T. Barkema, Phys. Rev. E 53, 393 (1996). A. Middleton and D.S. Fisher, Phys. Rev. B 65, 134411 (2002).

[22] G. Parisi, F. Ricci-Tersenghi and J.J Ruiz-Lorenzo, Eur. Phys. J. B 11, 317 (1999).

[23] K.H. Fischer and J.A. Hertz, Spin Glasses (Cambridge University Press, 1991).

[24] J.R.L. de Almeida and D.J. Thouless, J. Phys. A 11, 983 (1978).

[25] D.S. Fisher and D.A. Huse, J. Phys. A 20, L1005 (1987).

[26] A. Montanari and G. Semerjian, J. Stat. Phys. 124, 103 (2006).

[27] C.M. Fortuin, P.W. Kasteleyn and J. Ginibre, Communications in Mathematical Physics 22, 89 (1971).

[28] M. Mézard and G. Parisi, Eur. Phys. J. B 20, 217 (2001).

[29] M. Mézard and A. Montanari, Information, Physics, and Computation (Oxford University Press, 2009).

[30] L. Zdeborová, Acta Physica Slovaca 59, 169 (2009).

[31] A. Pagnani, G. Parisi and M. Ratieville, Phys. Rev. E 68, 046706 (2003).

[32] In a scenario known as dynamical replica symmetry breaking (see for instance [26]) the spin glass susceptibility does not diverge. This scenario is, however, impossible in finite dimensional systems. We also checked that it does not occur for the RFIM on the Bethe lattice. Beside, the "transition" into this phase is only a topological one in the free energy landscape, with no associated singularity in the free energy. 